\title{
Placental mesenchymal dysplasia
}

\section{Gujral Kanwal $^{1 *}$, Dimri Nandita ${ }^{2}$, Bhalla Sunita ${ }^{3}$, Mansukhani Chandra1 ${ }^{1}$, Nayar Sakshi ${ }^{4}$}

\author{
${ }^{1}$ Institute of Obstetrics and Gynecology, Sir Ganga Ram Hospital, New Delhi, India \\ ${ }^{2}$ Department of Fetal Medicine, Sir Ganga Ram Hospital, New Delhi, India \\ ${ }^{3}$ Department of Histopathology, Sir Ganga Ram Hospital, New Delhi, India \\ ${ }^{4}$ Department of Obstetrics and Gynecology, Lady Hardinge Medical College and Smt. S.K. Hospital, New Delhi, India
}

Received: 24 January 2017

Revised: 31 January 2017

Accepted: 02 March 2017

*Correspondence:

Dr. Gujral Kanwal,

E-mail: kgg_in@yahoo.com

Copyright: () the author(s), publisher and licensee Medip Academy. This is an open-access article distributed under the terms of the Creative Commons Attribution Non-Commercial License, which permits unrestricted non-commercial use, distribution, and reproduction in any medium, provided the original work is properly cited.

\section{ABSTRACT}

Placental Mesenchymal Dysplasia (PMD) is a rarely encountered placental lesion often associated with high fetal mortality and morbidity. We report here a case of PMD with a favourable outcome. The salient features of diagnosis, management and literature review are discussed.

Keywords: Cystic Placenta, Placental Mesenchymal Dysplasia

\section{INTRODUCTION}

Placental mesenchymal dysplasia (PMD) is a rare benign placental lesion characterized by placentomegaly and grape like vesicles. PMD closely resembles a molar pregnancy on sonography and gross placental examination but unlike molar pregnancy there is absence of trophoblast hyperplasia on histopathology. The lesion was first described by Moscoso in 1991. The true incidence of PMD is unknown but estimated to be at $0.02 \% .^{2}$

It could be possible that future may see a rise in incidence because of recent awareness of the condition. About $20 \%$ of fetuses with PMD are associated with Beckwith Wiedemann syndrome comprising of macrosomia, macroglossia, visceromegaly and omphalocele. ${ }^{3}$ Foetus typically is normal in majority of cases. PMD is often associated with adverse perinatal outcomes. We describe here a case of PMD with a favourable outcome along with the current understanding of pathogenesis, diagnosis, management and review of literature.

\section{CASE HISTORY}

Mrs ABC, 26 years old, G2A1 presented to us at 25 weeks of pregnancy for confinement. This was a spontaneous conception, pregnancy was dated in first trimester, all antenatal investigations including aneuploidy screen were normal.

Ultrasound at 20 weeks and subsequent at our hospital showed (Figure 1), fetal growth at $<10^{\text {th }}$ centile with normal fetal anatomy. However, placenta was occupying entire bulk of uterus with multiple cystic areas focally distributed. Doppler revealed a stained-glass appearance that is cystic spaces devoid of blood flow and surrounding parenchyma with increased blood flow and dilated chorionic vessels.

Keeping in mind the differential diagnosis of large cystic placenta, workup included serum Beta human chorionic gonadotrophins ( $\beta \mathrm{hCG})$, serum alpha fetoproteins (AFP), venereal disease research laboratory (VDRL), Toxoplasmosis, rubella, cytomegalovirus and herpes simplex virus (TORCH IgG and IgM) and amniocentesis 
for fetal karyotyping - Fluorescence in situ hybridization (FISH) and culture. The results were $\beta$ hCG - 1667, AFP10.5 MoM, VDRL, TORCH IgM and IgG negative, FISH-no aneuploidy, culture normal Karyotype. So, by process of exclusion, provisional diagnosis of placental mesenchymal dysplasia was made.

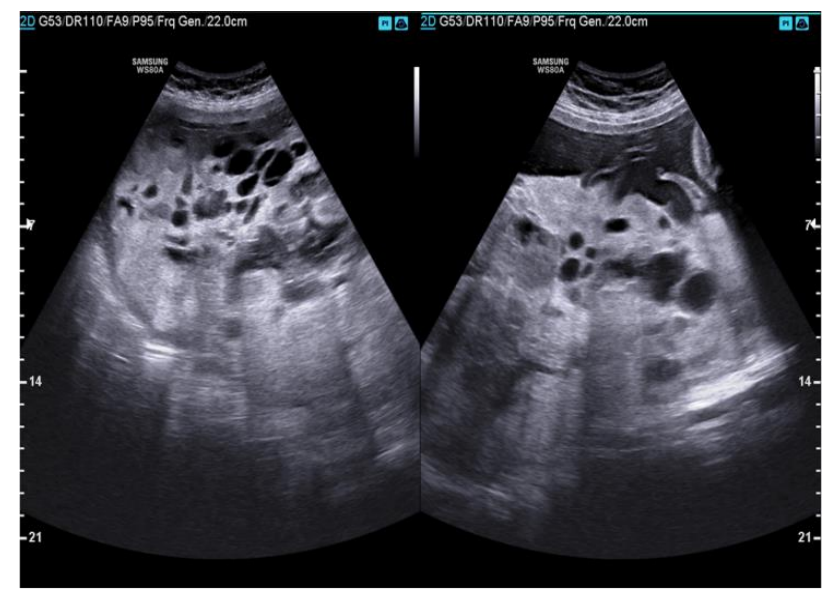

Figure 1: PMD ultrasound scan.

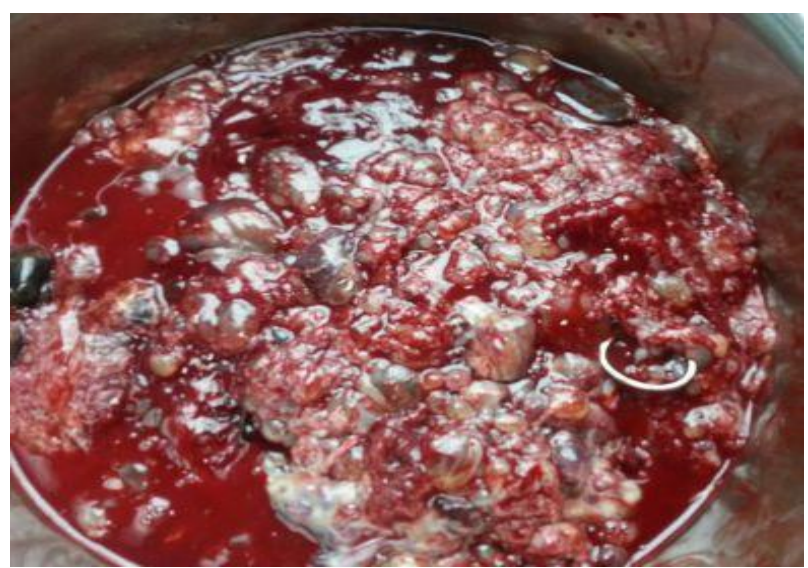

Figure 2: Gross specimen of placenta showing vesicles.

Patient was admitted with bleeding per vaginum at 28 weeks of gestation, Dexamethasone was given for enhancing fetal lung maturity.

Ultrasound at this point of time showed a collection of $12 \times 3 \times 8 \mathrm{~cm}$ near fetal site of placenta, fetal weight 893 gms, activity normal. Placenta had further enlarged filling almost $2 / 3$ of uterine cavity. Patient went into labor after 2 days, magnesium sulphate $\left(\mathrm{MgSO}_{4}\right)$ for fetal neuroprotection was given. After about 8 hours she had a small bout of fresh bleeding, decision for caesarean section was taken. A female fetus weighing $930 \mathrm{gms}$ with APGAR score $8 / 10$ was born. Placenta was massively large $(27 \times 20 \times 9 \mathrm{~cm})$ and full of vesicles. Placental weight was 2065 gms (Figure 2).

Patient had mild atonic postpartum hemorrhage which responded to oxytocics. New born was shifted to neonatal intensive care unit. Baby developed respiratory distress, needed ventilation, parenteral nutrition, and other supports. Discharged on day 65 at birth weight of 1810 grams. Mother had an uneventful postpartum period. $\beta$ hCG became normal at four weeks postpartum. Placental histopathology was confirmatory of PMD (Figure 3 and Figure 4).

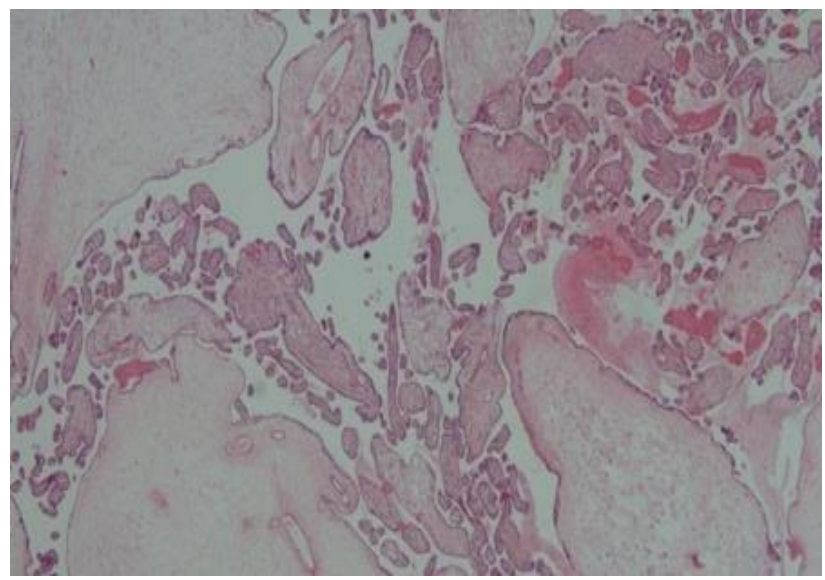

Figure 3: Enlarged chorionic villi with hydropic change and central cistern formation whereas many of the villi are unremarkable. There is no trophoblastic proliferation.

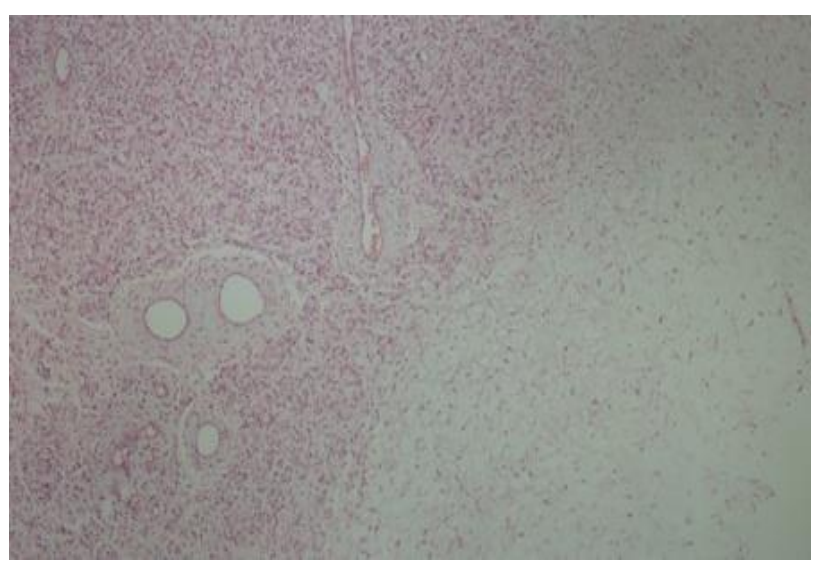

Figure 4: Dilated thick walled vessels with fibromuscular hyperplasia.

\section{DISCUSSION}

MOSCOS (1) in 1991 first described the lesion as stem cell villous hyperplasia, ultrasound picture resembling a partial mole and raised AFP. The current reported incidence is $0.02 \%$ with female to male ratio as $3.6: 1 .^{2,3}$ Till date about 100 cases have been reported. ${ }^{4}$

\section{Etiology}

The postulated etiologies are - congenital malformation of mesoderm, androgenetic/biparental mosaicism with endo reduplication of haploid paternal genome and molecular disruption of chromosome 11, p15-5 an association seen with Beckwith Wiedemann syndrome. ${ }^{5-7}$ 


\section{Diagnosis}

Ultrasound is the main stay in diagnosis. The suggested ultrasonic features include large thick placenta, multiple cystic echolucent areas like vesicles, focally distributed with areas of normally appearing echogenic parenchyma in between, dilated chorionic vessels which may show thrombosis. Doppler has stained glass appearance, echolucent cysts devoid of blood flow and surrounding parenchyma with normal blood flow. Fetal karyotyping is usually normal. Only one case of triploidy has been reported with PMD. ${ }^{3}$ Other contributory diagnostic features are raised AFP and normal to mildly increased BhCG. ${ }^{1,3}$

\section{Differential diagnosis}

Partial Hydatiform mole (elevated hCG, triploid fetus), complete hydatiform mole (absent or malformed fetus, markedly elevated hCG), choriangioma, intervillous hematoma, non specific hydropic changes (all have specific ultrasound features). Perinatal infections (syphilis, TORCH complex) are other differentials. Linda et al, in an excellently written review on PMD have reported following gross and histopathological features of placenta. $^{4}$

\section{Gross examination}

Typically, a very large placenta resembling a mole, cut section exhibits large grape like villi along with normal placental parenchyma in between.

\section{Histopathology features}

- Mixture of normal and abnormal villi

- Enlarged proximal stem villi with edematous/myxomatous stroma with cistern formation and thick walled peripheral blood vessels

- Distal villi have increased capillaries, chorioangiomata, chorioangiosis, and increased nucleated fetal erythrocytes.

- Normal villous trophoblast unlike molar pregnancy which has hyperplasia of trophoblast.

- Fetal thrombotic vasculopathy (FTV) - thrombi within the large, dilated chorionic vessels, vaso obliterative changes in stem villous vessels, and avascular villi or villous stromal vascular karyorrhexis in the distal villi

- Abnormal immunohisto chemical expression, P57 KIP2

\section{Fetal risks}

Anomalies include Beckwith Wiedemann syndrome (BWD) which is the commonest association seen in upto $20 \%$ of cases. ${ }^{3}$ BWD and PMD share a common etiology because they have similar spectrum of phenotype changeat one end phenotyping changes limited to placenta and at the other end affecting both the fetus and the placenta. ${ }^{8}$
Other associated anomalies include-congenital hemangiomas, hepatic mesenchymal hamartoma, pulmonary hamartoma, omphalocele, and gastroschisis. Intrauterine growth restriction (IUGR), intrauterine fetal death (IUFD) and preterm labour (PTL) are also associated with PMD. The cause of IUGR and IUFD is chronic fetal hypoxia secondary to FTV. Occasionally rupture of chorionic vessel can also lead to fetal death. ${ }^{9,10}$ Maternal risks include polyamnios and preeclampsia. ${ }^{11,12}$ Pregnancy Surveillance involves a careful search for anomalies, monitoring for fetal growth, PTL warning signs, and maternal blood pressure. $\beta$ hCG becomes normal within few weeks of delivery. Follow up with $\beta$ hCG like a molar pregnancy is not recommended.

Nayeri et al in a systematic review data base from 1991 to 2011 have reported following associations with PMDcystic placenta $80 \%$, enlarged thick placenta $50 \%$, dilated chorionic vessels $16 \%$, increased $\beta$ hCG $38 \%$, increased AFP $70 \%$, fetal anomalies 38\%, BWD syndrome $19 \%$, neonatal hepatic tumor $17 \%$, neonatal anemia and or thrombocytopenia 5\%, IUGR 33\%, IUFD 13\%, preterm delivery $52 \%$, preeclampsia, eclampsia, HELLP, and gestational hypertension 9\%. ${ }^{13}$ Pregnancy was uncomplicated only in $9 \%$ of cases.

\section{CONCLUSION}

PMD is an infrequently encountered placenta lesion. The entity should be a part of differential diagnosis of a cystic large placenta.

\section{Funding: No funding sources \\ Conflict of interest: None declared \\ Ethical approval: Not required}

\section{REFERENCES}

1. Moscoso G, Jauniaux E, Hustin J. Placental vascular anomaly with diffuse mesenchymal stem villous hyperplasia. A new clinico-pathological entity? Pathol Res Pract. 1991;187:324-8.

2. Arizawa M, Nakayama M. Suspected involvement of the $\mathrm{X}$ chromosome inplacental mesenchymal dysplasia. Congenit Anom. 2002;42:309-17.

3. Cohen MC, Roper EC, Sebire NJ, Stanek J, Anumba DOC. Placental mesenchymal dysplasia associated with fetal aneuploidy. Prenat Diagn. 2005;25:187-92.

4. Ernst LM. Placental mesenchymal dysplasia. J Fetal Med. 2015;2(3):127-33.

5. Chen CP, Chern SR, Wang TY, Haung MC, Chaung CY. Pregnancy with concomitant chorangioma and placental vascular malformation with mesenchymal hyperplasia. Hum Reprod. 1997;12:2553-6.

6. Kaiser Rogers KA, McFadden DE, Livasy CA, Dansereau J, Jiang R, Knops JF et al. Androgenetic/biparental mosaicism causes placental mesenchymal dysplasia. J Med Genet. 2006;43:187-92. 
7. Maher ER, Reik W. Bechwith-Wiedemann syndrome: imprinting in clusters revisited. J Clin Invest. 2000;105:247-52.

8. Zahida P, Fraser R, Jeffery LK, Karen ST. Placental mesenchymal dysplasia. Arch Pathol Lab Med. 2007;131:131-7.

9. Kuwabara Y, Shima Y, Araki T, Shin S. Mesenchymal stem villous hyperplasia of the placenta and fetal growth restriction. Obstet Gynecol. 2001;98:940-3.

10. Pham T, Steele J, Stayboldt C, Chan L, Benirschke K. Placental mesenchymal dysplasia is associated with high rates of intrauterine growth restriction and fetal demise: a report of 11 new cases and a review of the literature. Am J Clin Pathol. 2006;126:67-78.
11. Cohen MM Jr. Beckwith Wiedemann syndrome: historical, clinicopathological, and etiopathogenetic perspectives. Pediatr Dev Pathol. 2005;8:287-304.

12. McCowan L, Becroft D. Beckwith Wiedemann syndrome, placental abnormalities, and gestational proteinuric hypertension. Obstet Gynecol. 1994;83:813-7.

13. Nayeri UA, West AB, Grossetta Nardini HK, Copels JA. Systematic review of sonographic findings of placental mesenchymal dysplasia and subsequent pregnancy outcome. Ultrasound Obstet Gynecol. 2013;41:366-74.

Cite this article as: Kanwal G, Nandita D, Sunita B, Chandra M, Sakshi N. Placental mesenchymal dysplasia. Int J Reprod Contracept Obstet Gynecol 2017;6:1664-7. 